\title{
AUGE MINERO Y DESINDUSTRIALIZACIÓN EN AMÉRICA LATINA
}

\author{
Edwin Torres Gómez* \\ Mauricio López González***
}

F $\mathrm{n}$ las últimas décadas, América Latina mostró tasas de crecimiendel ciclo económico, registró tasas muy superiores a los de otros continentes e incluso a la de los países industrializados. De acuerdo con la Cepal, su crecimiento promedio entre 1990 y 2003 fue del 2,6\%, entre 2004 y 2008 del 5,2\% y entre 2009 y 2014 del 2,7\%. Desde 1990 también se observan cambios muy visibles en la participación de los sectores minero e industrial en el producto total. El sector minero pasó del 3,7\% del рів en el periodo 1990-2003, al 6,2\% en el periodo 2004-2014. Y el sector industrial se redujo del 16,5\%, al 14,3\%1. Esta situación obedece al aumento de precios de los bienes básicos, la mayor inversión extranjera en la región y el auge de la producción de bienes mineros en varios países, algunos de ellos con altos niveles de producción, así como a la reducción sistemática de la producción y las exportaciones de bienes manufacturados.

Este artículo explora la relación entre el aumento de la producción minera y la reducción de la producción industrial en varios países latinoamericanos e intenta verificar la existencia de enfermedad holandesa, tomando las exportaciones de ambos sectores como proxies

* Estudiante de Doctorado en Economía, profesor de la Universidad de Antioquia. Medellín, Colombia, [esteban.torres@udea.edu.co].

** Estudiante de Doctorado en Dirección de Empresas, profesor de la Universidad de Antioquia. Medellín, Colombia, [mauricio.lopez@udea.edu.co]. Este artículo es un resultado de la línea de investigación en comercio exterior del Grupo de Macroeconomía Aplicada de la Universidad de Antioquia. Fecha de recepción: 19-08-2016, fecha de modificación: 11-08-2017, fecha de aceptación: 04-09-2017. Sugerencia de citación: Torres G., E. y López G., M. (2017). Auge minero y desindustrialización en América Latina, Revista de Economía Institucional, 19(37), 133-146. Dor: https://doi.org/10.18601/01245996.v19n37.07

1 Los datos corresponden al promedio del total de América Latina; ver [http://estadisticas.cepal.org/cepalstat/weB_CePAlstat/Portada.asp]. 
de la producción y la comercialización. Se pretende identificar un patrón de comportamiento de la producción de diversos sectores cuando uno de ellos está en expansión, y elaborar lo que llamamos curva de sustitución intersectorial.

$\mathrm{E} 1$ artículo consta de cuatro secciones. La primera describe el marco teórico. La segunda muestra la evolución de algunos indicadores económicos de la región y analiza las brechas entre producción industrial y minera. La tercera presenta el modelo de enfermedad holandesa que se emplea para verificar algunas hipótesis y correlaciones. La última expone las conclusiones.

\section{MARCO CONCEPTUAL}

El impacto negativo de la producción de bienes mineros en la producción de otros bienes transables ha sido objeto de preocupación y análisis en la literatura económica, que lo denomina "enfermedad holandesa". Por ejemplo, el descubrimiento de un bien transable como el petróleo puede generar una apreciación de la tasa de cambio real (Baldwin, 1966) e indirectamente un desplazamiento de la producción de otros bienes y servicios (Krugman, 1987).

A este respecto se han hecho numerosos trabajos teóricos y empíricos. Así, por ejemplo, Corden y Neary (1982) es un estudio pionero sobre la relación entre el auge de un producto y la desindustrialización, Hamilton (1983) analiza la relación entre el petróleo y la economía estadounidense, Rudd (1996) analiza los efectos de la enfermedad holandesa en países industrializados y no industrializados, Spatafora y Warner (1999) los efectos de la exportación de petróleo sobre la economía de los países en desarrollo. Algunos trabajos emplean modelos relativamente sofisticados, como el de Stijns (2003), que elabora un modelo gravitacional para la economía mundial y encuentra una relación directa entre el auge de las exportaciones de un recurso natural y la desindustrialización ${ }^{2}$.

Los primeros hallazgos teóricos fueron obtenidos por Corden y Neary (1982) y Neary y Van Wijnbergen (1986), para una economía abierta pequeña con pocas restricciones a los flujos de capital. Su modelo consta de tres bienes, dos de ellos transables, de los cuales uno es industrial y el otro, del sector primario, experimenta un auge. El modelo identifica dos efectos del auge exportador sobre los demás sectores económicos, tanto transables como no transables.

\footnotetext{
2 Aunque la desindustrialización no es tan solo resultado de la enfermedad holandesa.
} 
E1 primero es el traslado de recursos productivos: el aumento exógeno del precio del producto que experimenta el auge exportador aumenta el producto marginal del trabajo en este bien. Esto atrae mano de obra y recursos financieros de los demás bienes y contrae el sector transable, donde se subutilizan sus factores de producción. Este traslado de recursos productivos eleva el precio de los bienes no transables porque su demanda excede a la producción. Y esto lleva a una apreciación de la tasa de cambio real (López et al., 2016).

$\mathrm{E} 1$ segundo es un efecto sobre el gasto: el auge exportador aumenta los ingresos domésticos y con ello la demanda de todos los bienes. Como el precio de los bienes transables se ajusta en el mercado internacional, este aumento del gasto eleva el precio relativo de los no transables, lo que aprecia la tasa de cambio real. En respuesta, la mano de obra se traslada de los sectores transables a los no transables, lo que contrae los sectores transables que no están en auge (ibíd.). En suma, se presenta un trade off que se manifiesta en una serie de consecuencias y cambios de algunas variables económicas:

- Apreciación de la tasa de cambio real.

-Aumento de precios de algunos bienes no transables con un efecto ambiguo sobre su producción (en algunos puede aumentar, en particular los que están asociados al sector en auge).

- Reducción de la producción y exportación de otros bienes transables, en especial de la industria.

- Movilidad de capital entre sectores.

La movilidad del capital se detecta en análisis dinámicos (Corden, 1984) porque el capital no cambia en un estado estacionario (Buffie, 1992). Diversos trabajos que analizan los movimientos del trabajo y el capital entre sectores muestran que el sector en auge, y algunos que tradicionalmente no son transables, se benefician con esos movimientos de factores.

El sector manufacturero es de suma importancia para las economías porque sus encadenamientos hacia adelante y hacia atrás son mayores que en el sector primario. En la producción industrial hay más división del trabajo y se genera mayor valor agregado que en la producción de bienes primarios (Hirschman, 1958; Seers, 1964; Baldwin, 1966). Además, cuanto más avanzado tecnológicamente es el sector industrial tiende a generar empleos de mejor calidad y se presenta una menor informalidad (Uribe y Ortiz, 2006).

Cuando se exporta una alta proporción de recursos naturales con respecto a las exportaciones totales, es decir, cuando la economía depende principalmente del sector primario, es posible que se estén 
presentando fenómenos asociados con políticas comerciales poco efectivas, bajas tasas de inversión privada y externa, bajo PIв per cápita, y bajo nivel de gasto público (Sachs y Warner, 1995: López et al., 2016). Como parece suceder en algunos países de América Latina.

Los términos de intercambio se recuperaron de la caída de los años ochenta y muestran una tendencia volátil, pero positiva. Así, la nueva estructura de la oferta y la demanda mundial de bienes podría modificar la tendencia de los precios de bienes primarios y productos básicos manufacturados a largo plazo, lo que debería tenerse en cuenta al diseñar instrumentos fiscales (Jiménez y Tromben, 2006).

Otros estudios presentan resultados complementarios. Allcott y Keniston (2014) encuentran que, en la economía norteamericana, el auge de un bien primario genera cierto nivel de aprendizaje que permite minimizar los efectos negativos sobre la industria, aunque haya aumentos de salarios y movilidad de mano de obra. De otro lado, Lartey et al. (2012) encuentran que, en un conjunto de países en vías de desarrollo, aumentos inusitados de las remesas tienen efectos negativos sobre los bienes no transables y la tasa de cambio.

Wikstrom y Osterberg (2003) evalúan el auge del aluminio en Jamaica y encuentran que, pese a la entrada de divisas y mayores inversiones, no hubo síntomas de enfermedad holandesa. En Ecuador, parte de la discusión se centró en el destino adecuado de los recursos públicos originados por la bonanza, para afectar lo menos posible a otros sectores transables. Fontaine (2013) propuso que, ante un auge inusitado, la inversión pública debía privilegiar el gasto en infraestructura (vías, bienes raíces, servicios públicos) o proteger sectores tradicionales que compiten con el petróleo (agricultura, industria y comercio).

Lanteri (2015) demuestra que en Argentina no hubo enfermedad holandesa con el auge del sector agropecuario entre 1993 y 2015, considerando las relaciones entre ese sector en expansión y el sector industrial. Al analizar el caso de Argentina, pero incluyendo a Brasil y Chile, se observa que la estructura productiva puede volverse irrecuperable cuando la difusión de la enfermedad holandesa se asocia al cierre de firmas del sector transable y a la pérdida de capital empresarial y humano ligado a esas empresas (Katz y Bernat, 2014).

En la literatura también figuran análisis que intentan relacionar la enfermedad holandesa con otras variables económicas o políticas. Isham et al. (2005), Robinson et al. (2006) y Mehlum et al. (2006) muestran la incidencia de un ciclo político en la explotación de recursos naturales que están en auge y el impacto negativo sobre los demás 
sectores, una vez la política económica los aísla. En otras palabras, la política pública tiende a favorecer sectores en auge en detrimento de otros sectores transables.

Este fenómeno también ha sido descrito teóricamente mediante el modelo del comercio exterior de Eli Heckscher y Bertil Ohlin, según el cual un choque positivo en el precio relativo de un bien, en una economía abierta, puede modificar el patrón exportador del país, al trasladar factores productivos y concentrarlos en la producción del bien o sector favorecido por el choque de precios relativos; un fenómeno que en teoría se conoce como teorema de Stolper-Samuelson.

\section{EXPORTACIONES Y TENDENCIAS DE LARGO PLAZO}

Los productos energéticos experimentaron una fuerte alza de precios entre 2000 y 2014, como muestra el índice de precios de la energía de la gráfica 1, que condensa los precios internacionales de petróleo, carbón y gas, entre otros. Este fuerte aumento del índice está asociado estrechamente a las fluctuaciones del precio del petróleo en ese periodo, que pasó de 10,4 dólares en diciembre de 1998 a 132,6 en julio de 2008) y mantuvo un alto nivel después de un brusco descenso (gráfica 2), lo cual provocó un auge internacional de las exportaciones de estos productos por el alza de sus precios internacionales.

\section{Gráfica 1}

Índice de precios de la energía

$2005=100$

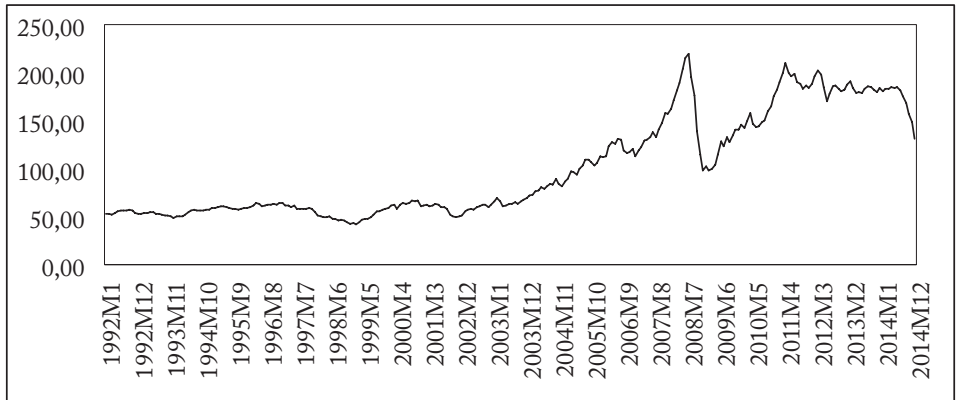

Fuente: FMI, elaboración propia.

El alza de precios aumentó las exportaciones de productos energéticos y mineros de los países latinoamericanos y elevó su participación en las exportaciones totales mientras que la participación de las exportaciones industriales en las exportaciones no mineras se mantuvo relativamente estable entre 1996 y 2014 (gráfica 3). De modo que 
la exportación de minerales ha ganado peso relativo con respecto a la exportación de bienes industriales, y el auge del sector minero no parece haber impulsado el crecimiento de otros sectores.

El análisis de las series de cada país confirma los resultados del modelo de Heckscher-Ohlin en algunos de estos países; el alza de los precios relativos de un sector transable provoca una redistribución de los recursos y modifica el patrón de especialización; un sector pierde participación en las exportaciones y aumenta la del sector en auge.

Gráfica 2

Precio internacional del petróleo en dólares

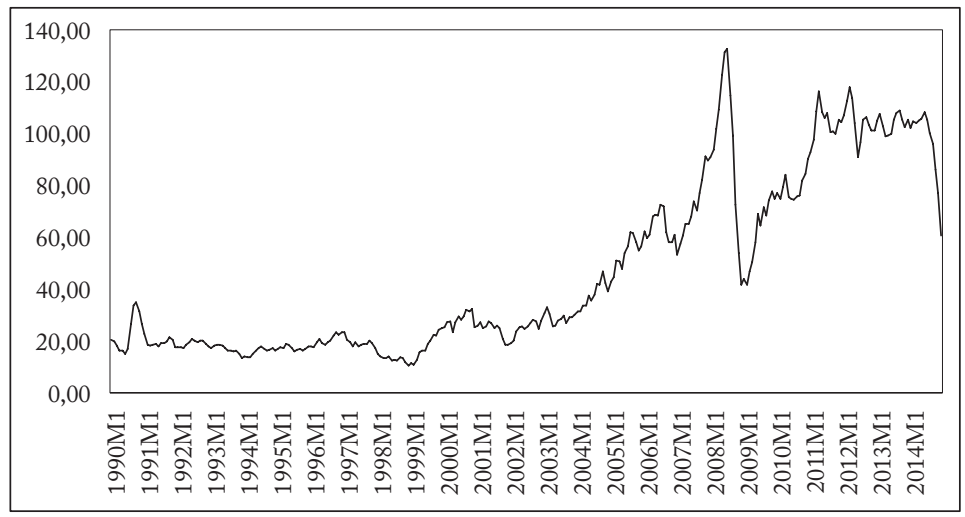

Fuente: FMI, elaboración propia.

Gráfica 3

Proporción de las exportaciones industriales y mineras, América Latina

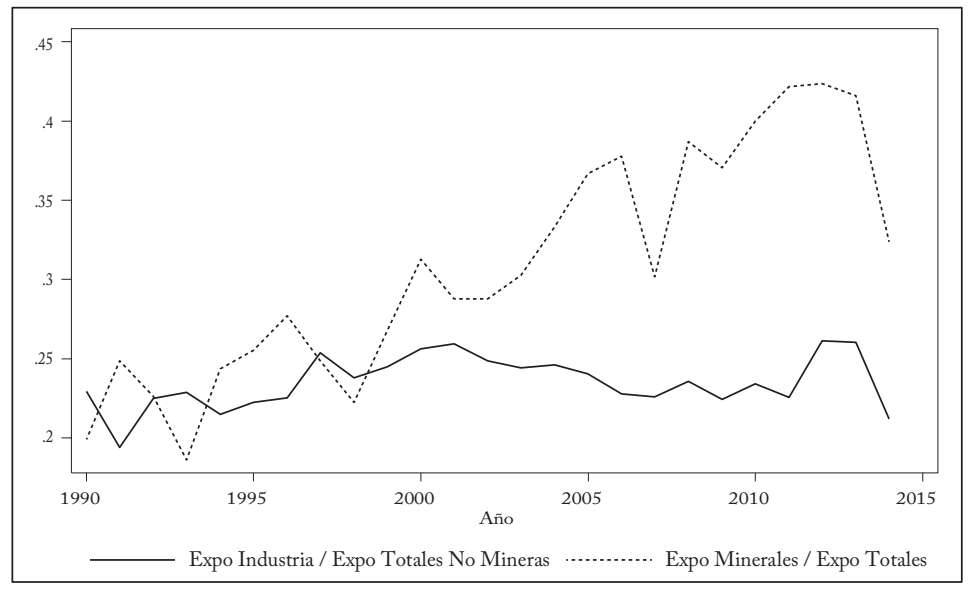

Fuente: UN Comtrade, elaboración propia. 
La gráfica 4 muestra la proporción de las exportaciones mineras e industriales de ocho países. El resultado mencionado es mucho más claro en Brasil, Colombia y Perú; en Argentina y México sucede lo contrario (cae la proporción de exportaciones mineras y aumenta la de las industriales). En Venezuela las exportaciones no mineras solo representan un 5\% del total; de modo que el aumento de la proporción de exportaciones industriales tiene que obedecer al crecimiento de alguna rama industrial.

Gráfica 4

Proporción de las exportaciones mineras e industriales, por país

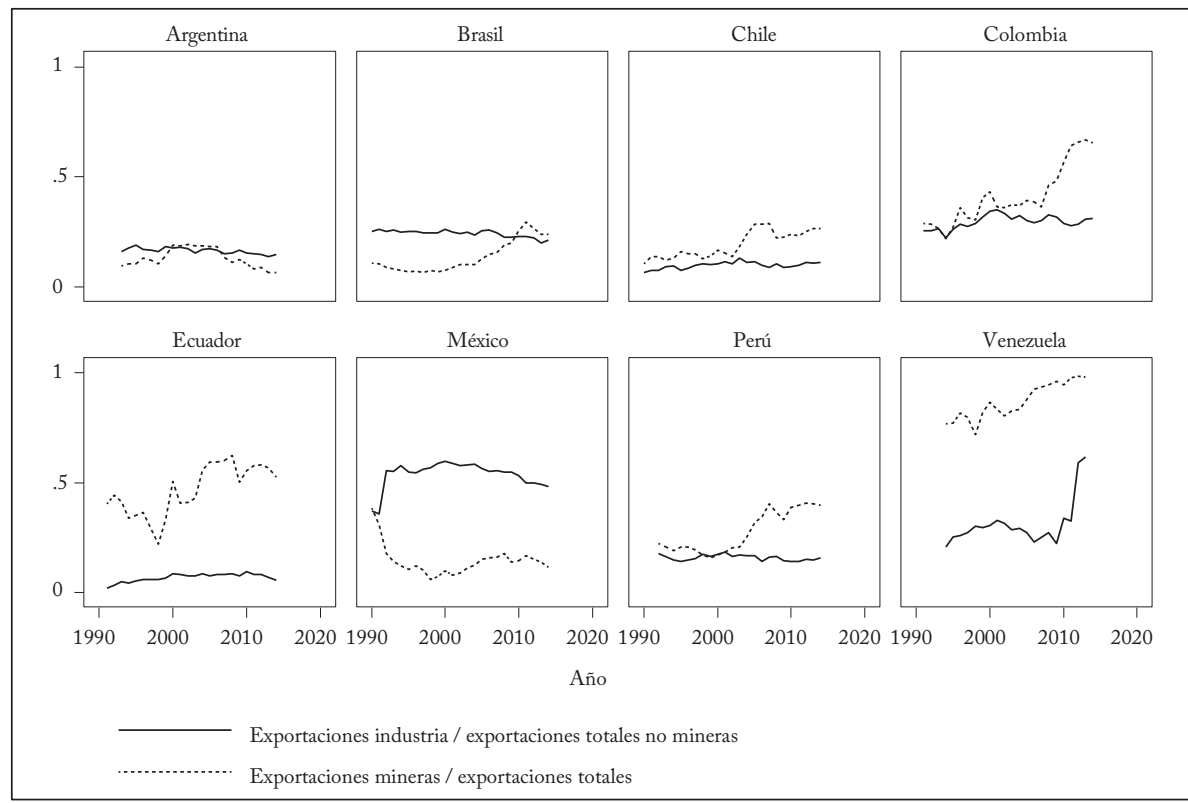

Fuente: UN Comtrade, elaboración propia.

Los resultados anteriores sugieren que el alza del precio internacional de los productos minerales afecta de manera diferente a los países exportadores, según el peso del sector en auge en las exportaciones totales y el tiempo que tarda la respuesta de los inversionistas para trasladar recursos de un sector a otro. Para analizar este aspecto, la gráfica 5 muestra la curva resultante de una regresión local polinómica que estima la correlación entre exportaciones industriales/ exportaciones no minerales y exportaciones minerales/exportaciones totales y sus intervalos de confianza respectivos. La llamamos "curva de sustitución intersectorial” (CSI) porque muestra el desplazamiento de las exportaciones de un sector por las de otro sector. 
La csi del conjunto de América Latina muestra que este desplazamiento tiene la forma de una campana. Al inicio, cuando empieza el auge de la exportación de minerales, y su participación es relativamente baja (es decir, la economía no depende de la minería) puede tener efectos que favorecen al sector industrial, pero luego de alcanzar un punto máximo la economía se vuelve tan dependiente de la minería que un auge de este sector perjudica a la industria.

Gráfica 5

Curva de sustitución intersectorial, América Latina

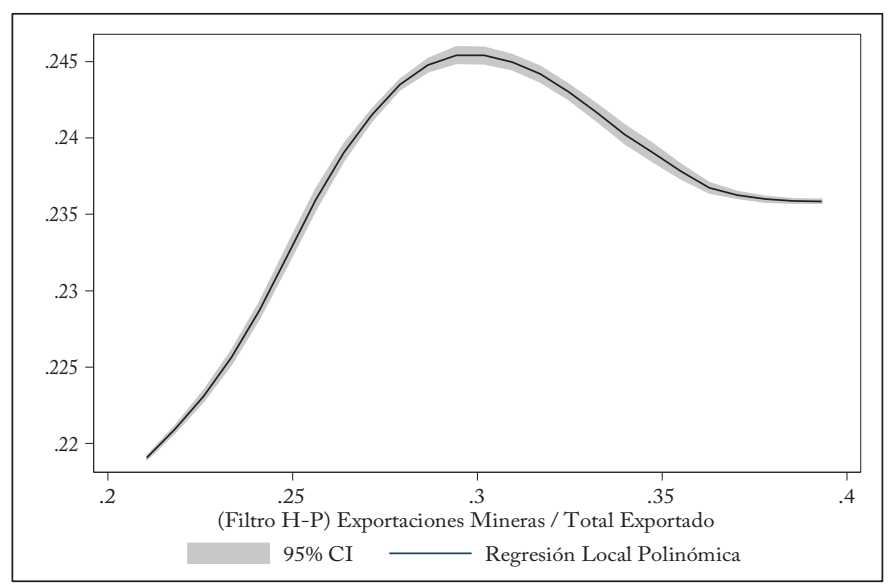

Fuente: UN Comtrade, elaboración propia.

Dada la heterogeneidad de las economías de los países latinoamericanos y considerando que todos dependen en mayor o menor proporción del sector minero, es de esperar que al observar la correlación de cada país por separado se sitúe en una parte distinta de la curva de sustitución intersectorial.

La gráfica 6 muestra los resultados de la csi para los mismos ocho países. La línea vertical, indica tanto la proporción de las exportaciones de minerales en 2014 como el lugar de la curva en que se encuentra cada país. Algunos países se sitúan en el tramo perjudicial de la csi (después del máximo, donde el auge minero reduce la proporción de las exportaciones industriales en las exportaciones no mineras): Brasil, Chile, Colombia y Perú. Ecuador y México también se sitúan en el tramo decreciente, pero en un lugar que no es tan perjudicial como el de los países anteriores. En Argentina, el crecimiento de las exportaciones mineras no tiene efectos perjudiciales sobre su industria. El caso de Venezuela es muy especial, como ya se mencionó, pues sus exportaciones no mineras solo representan el 5\% del total. 
Estos resultados son consistentes con la desindustrialización asociada a la enfermedad holandesa, cuyos efectos son de mayor o menor magnitud según el país, pero se observan en toda la región. Cabe entonces señalar que la muestra de países corresponde a economías grandes en términos de PIB, aunque no difieren en el nivel de producción y comercialización de bienes mineros e industriales, así como en el tamaño del sector minero. No obstante, la validez de los resultados, en particular el traslado de recursos a los sectores en auge, no depende de estas diferencias; solo afecta la magnitud de los efectos.

Gráfica 6

Tramos de la curva de sustitución intersectorial por país

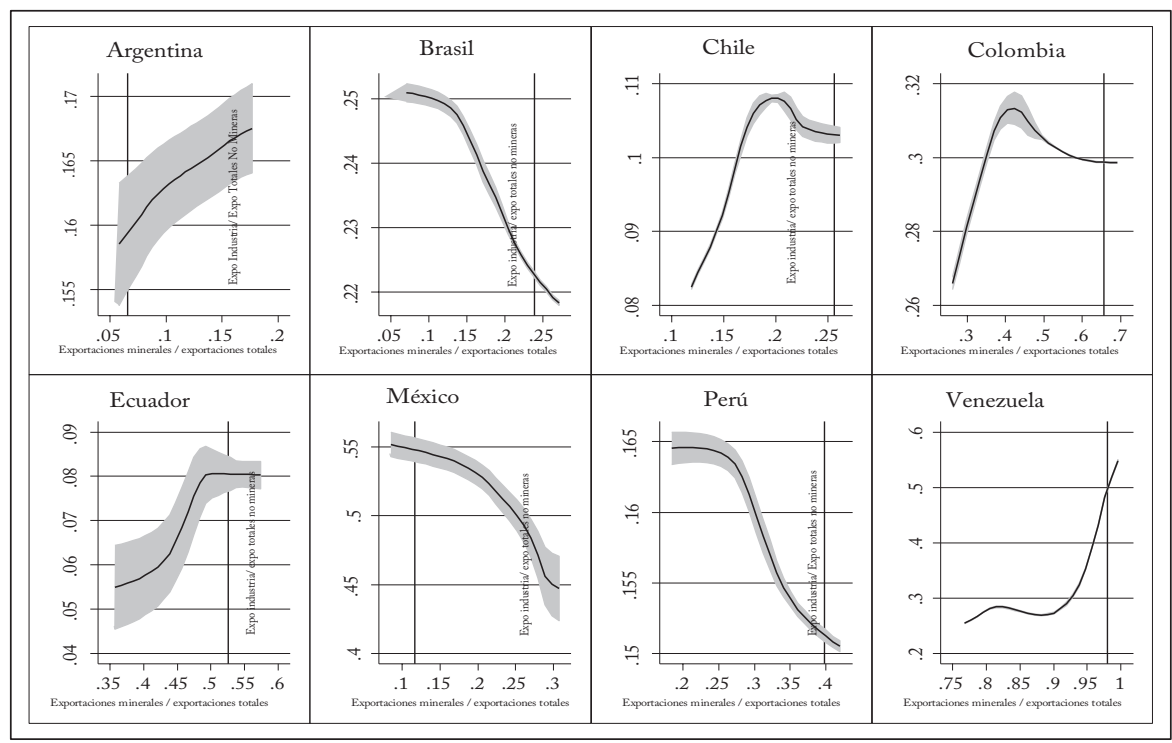

Fuente: UN Comtrade, elaboración propia.

\section{MODELACIÓN Y RESULTADOS}

La estrategia empírica consiste en representar la enfermedad holandesa mediante un modelo de panel de datos con efectos fijos donde la variable dependiente es la proporción entre exportaciones industriales y exportaciones no mineras, para el grupo de los ocho países ya mencionados y el periodo 1990-2014. Rudd (1996) propone estimar un modelo econométrico de la forma siguiente:

Declive del sector manufacturero $=f$ (efecto gasto, efecto traslado de recursos) 
Siguiendo a Nyatepe-Coo (1994), la proporción mencionada evita la posibilidad de que el cociente disminuya por el simple crecimiento de la minería. El modelo que se estima es entonces:

$Y_{i t}=\beta_{0}+\beta_{1} X_{1 i t}+\beta_{2} X_{2 i t}+\beta_{3} X_{3 i t}+\varepsilon$

Los subíndices $t$ e $i$ indican el año y el país, respectivamente. E1 cuadro 1 describe la variable dependiente y el vector de variables dependientes.

Cuadro 1

Descripción de las variables

\begin{tabular}{ll}
\hline Notación & Variable \\
\hline$Y$ & Exportaciones industriales/Exportaciones no mineras \\
$X_{1 i t}$ & Exportaciones minerales/Exportaciones totales \\
$X_{2 i t}$ & (Exportaciones minerales/Exportaciones totales) ${ }^{2}$ \\
$X_{3 i t}$ & PIB per cápita \\
$X_{4 i t}$ & Tasa de cambio \\
\hline
\end{tabular}

Estas variables se pueden clasificar conforme a los efectos de la enfermedad holandesa: el efecto traslado de recursos y el efecto gasto. E1 primero incluye la proporción entre exportaciones mineras y exportaciones totales y esa proporción al cuadrado; y el efecto gasto, el рів per cápita y la tasa de cambio. El modelo se estima para varias ramas de la industria, con el fin de analizar cómo las afecta dicha enfermedad (cuadro 2).

Cuadro 2

Estimaciones del modelo por ramas industriales

\begin{tabular}{lccccc}
\hline Variables & $(1)$ & $(2)$ & $(3)$ & $(4)$ & $(5)$ \\
\hline Exp. mineras/Exp. totales & $-0.189^{* *}$ & $0.175^{* * * *}$ & $-0.189^{* * * *}$ & $-0.330^{* * * *}$ & $-0.533^{* * * *}$ \\
& $(0.086)$ & $(0.023)$ & $(0.028)$ & $(0.060)$ & $(0.095)$ \\
(Exp. mineras/Exp. totales) & $0.446^{* * *}$ & $-0.179^{* * * *}$ & $0.075^{* * *}$ & $0.249^{* * * *}$ & $0.592^{* * * *}$ \\
& $(0.087)$ & $(0.023)$ & $(0.028)$ & $(0.060)$ & $(0.095)$ \\
PIB per cápita & 0.000 & 0.001 & $-0.007^{* * * *}$ & 0.003 & -0.003 \\
& $(0.003)$ & $(0.001)$ & $(0.001)$ & $(0.002)$ & $(0.004)$ \\
Tasa de cambio & -0.001 & $0.001^{* *}$ & $0.001^{* * *}$ & $0.001^{*}$ & $0.002^{* * *}$ \\
& $(0.001)$ & $(0.000)$ & $(0.000)$ & $(0.001)$ & $(0.001)$ \\
Constante & $0.055^{* * *}$ & 0.006 & $0.111^{* * *}$ & $0.120^{* * *}$ & $0.292^{* * * *}$ \\
& $(0.019)$ & $(0.005)$ & $(0.006)$ & $(0.013)$ & $(0.021)$ \\
Observaciones & 177 & 177 & 177 & 177 & 177 \\
$\mathrm{R}^{2}$ & 0.248 & 0.302 & 0.516 & 0.173 & 0.205 \\
Número de países & 8 & 8 & 8 & 8 & 8 \\
\hline
\end{tabular}

Nota: La variable dependiente es Exportaciones industriales/Exportaciones no mineras totales. (1) Industria química, (2) Plásticos y manufacturas de plásticos, (3) Industria textil, (4) Maquinaria y equipos eléctricos y electrónicos, y (5) Industrial total. Los errores estándar van entre paréntesis; ${ }^{*} \mathrm{p}<0.1,{ }^{* *} \mathrm{p}<0.05,{ }^{* * *} \mathrm{p}<0.01$. 
Al contrastar la hipótesis de que existe desindustrialización a causa del auge internacional del sector minero se encuentra que la variable independiente (columna 5) tienen una correlación negativa y significativa con la proporción "Exportaciones mineras/Exportaciones totales", y la relación entre estas dos variables es una función convexa, es decir, el conjunto de la industria latinoamericana se sitúa en el lado derecho de la curva de sustitución intersectorial (el tramo de efectos perjudiciales cuando hay auge minero).

Las columnas 1, 2, 3 y 4 del cuadro 2 muestran los resultados de las regresiones de cada rama industrial. Las industrias química, textil, de maquinaria y equipos eléctricos y electrónicos se sitúan en el mismo tramo que el conjunto de la industria, con un efecto semejante, y coeficientes negativos y estadísticamente significativos. En la rama de plásticos y manufacturas de plástico (columna 2) la correlación es positiva y significativa, y el coeficiente de la proporción elevada al cuadrado es negativo, de modo que se sitúa en el tramo izquierdo de la curva y aún no ha sido perjudicado por el auge del sector minero, aunque la concavidad de la función indica que llegará a un máximo a partir del cual la participación de sus exportaciones en el total de exportaciones no mineras empezará a disminuir.

De acuerdo con la función resultante, ese nivel máximo corresponde, ceteris paribus, al punto $X^{*}=0,175 / 2(0,179)$, es decir $X^{*}=0,488$; de modo que si las demás condiciones de la economía se mantienen constantes, a partir de una proporción entre exportaciones mineras y exportaciones totales del 48,8\%, el sector de plásticos y manufacturas de plástico se verá perjudicado ${ }^{3}$. En 2014 esa proporción era del 6,5\% en Argentina, del 23,8\% en Brasil, del 26,6\% en Chile, del $11.1 \%$ en México y del 39,8\% en Perú, es decir, aún no había efectos perjudiciales en este sector. En cambio, en Colombia, Ecuador y Venezuela era del 65,6\%, del 52,6\% y del 98,0, respectivamente.

Los coeficientes del PIB per cápita no resultaron estadísticamente significativos, salvo en la industria textil que presenta una correlación negativa entre el crecimiento del PIв per cápita y su proporción de exportaciones. El coeficiente de la tasa de cambio resultó siempre positivo y significativo, de modo que una moneda depreciada con respecto al dólar favorece la exportación de productos industriales. En suma, los resultados indican que América Latina padece la enfermedad holandesa.

\footnotetext{
${ }^{3}$ Este valor se calcula derivando la función estimada en la columna 2 del cuadro 2 con respecto a la relación "Exportaciones mineras/Exportaciones totales" e igualando a 0 .
} 
Los modelos fueron sometidos a la prueba de Wooldridge para verificar la existencia de autocorrelación, y a la prueba de Wald para verificar la existencia de heteroscedasticidad, sin que se encontrara ese tipo de problemas. La prueba de Hausman indicó que lo más adecuado era utilizar el modelo de efectos fijos, y así se estimó; de modo que los ejercicios son consistentes y los resultados son confiables.

A diferencia del modelo tradicional que supone pleno empleo y rigidez de precios y salarios, nuestro ejercicio no requería esos supuestos porque la curva de sustitución intersectorial se construye a partir de la evidencia empírica, y porque se reconocen las diferencias de productividad tanto en los sectores y en las economías como en los precios y salarios de los diversos sectores.

\section{CONCLUSIONES}

Este trabajo identifica los mecanismos a través de los cuales el aumento de la exportación de productos mineros, al superar cierto umbral, reduce las exportaciones de bienes industriales, un efecto que llamamos curva de sustitución intersectorial; y constituye su principal aporte.

Ese umbral es determinado por las características de cada economía: capacidad de producción y exportación de bienes mineros e industriales, niveles de productividad y competitividad y contexto macroeconómico. La csi identifica el estado de las exportaciones mineras e industriales, y la distancia frente a problemas asociados con la enfermedad holandesa.

Se encuentra que el nivel de exportaciones mineras de América Latina ha llegado a un punto en que empieza a perjudicar las exportaciones industriales. En otras palabras, como un todo, la región se halla en una situación que requiere urgentemente promover la producción y la exportación de bienes industriales. Y para ello se requieren sólidas políticas de industrialización.

El trabajo es novedoso en cuanto el análisis parte de los componentes transitorios de las exportaciones de bienes mineros e industriales para aislar el efecto del ciclo económico sobre las exportaciones, haciendo estimaciones con efectos fijos.

En cuanto a los países particulares, el trabajo determina la ubicación de las economías analizadas en la csi, y esto hace posible determinar su estado y la sustitución entre sectores que han de soportar un auge del sector minero. En el caso de Argentina, el análisis se podría complementar considerando el sector agrícola en lugar del minero. 
Los resultados para Brasil, Chile, Perú y Colombia concuerdan con los de otros autores sobre la existencia de síntomas de enfermedad holandesa provocados por la expansión sostenida del sector minero. Si bien los indicios varían, se concentran en aspectos relacionados con la tasa de cambio, el traslado de inversiones entre sectores transables y el aumento de salarios en sectores específicos.

E1 trabajo deja abiertos, en particular, dos temas de futura investigación. El primero, identificar los subsectores mineros e industriales que se ven afectados, en cada economía, cuando hay un auge del sector minero. Pues aunque este trabajo distingue cuatro subsectores y el total industrial, analiza en forma agregada la producción y las exportaciones mineras. El segundo es examinar los efectos de la política fiscal. Puesto que es posible atenuar los efectos de la enfermedad holandesa con medidas fiscales contracíclicas, cuyo impacto es diferente según el país.

\section{REFERENCIAS BIBLIOGRÁFICAS}

1. Allcott, H. y Keniston, D. (2014). Dutch disease or agglomeration? The local economic effects of natural resource auges in modern America. NBER working paper 20508. [http://www.nber.org/papers/ w20508.pdf].

2. Baldwin, R. (1966). Economic development and export growth: A study of Northern Rhodesia, 1920-1960. Berkeley y Los Angeles, cA: University of California Press.

3. Buffie, E. F. (1992). On the condition for export-led growth. Canadian Journal of Economics, 25(1), 211-225.

4. Corden, W. M. (1984). Booming sector and Dutch disease economics: Survey and consolidation. Oxford Economic Papers, 36(3), 359-380.

5. Corden, W. M. y Neary, P. (1982). Booming sector and de-industrialization in a small open economy. Economic Journal, 92(368), 825-848.

6. Fontaine, G. (2002). Sobre bonanzas y dependencia: petróleo y enfermedad holandesa en el Ecuador. Íconos. Revista de Ciencias Sociales, (13), 102-110.

7. Hamilton, J. (1983). Oil and the macroeconomy since World War II. Journal of Political Economy, 91(2), 228-248.

8. Hirschman, A. (1958). The strategy of economic development. New Haven, Conn. Yale University Press.

9. Isham, J.; Woolcock, M. et al. (2005). The varieties of resource experience: Natural resource export structures and the political economy of economic growth. The World Bank Economic Review, 19(2), 141-174.

10. Jiménez, J. P. y Tromben, V. (2006). Política fiscal y bonanza: impacto del aumento de los precios de los productos no renovables en América Latina y el Caribe. Revista de la Cepal, (90), 61-86.

11. Katz, J. y Bernat, G. (2013). Dilemas de política económica en América Latina: evidencia para Argentina, Brasil y Chile. Revista de Economia Politica de Buenos Aires, 7(12), 73-104. 
12. Krugman, P. (1987). The narrow moving band, the Dutch disease, and the competitive consequences of Mrs. Thatcher: Notes on trade in the presence of dynamic scale economies. Journal of Development Economics, 27(1), 41-55.

13. Lanteri, L. (2015). Efectos de la enfermedad holandesa ('Dutch disease'). Alguna evidencia para Argentina. Revista de Economía del Rosario, 18(2), 187-209.

14. Lartey, E. K.; Mandelman, F. S. et al. (2012). Remittances, exchange rate regimes and the Dutch disease: A panel data analysis. Review of International Economics, 20(2), 377-395.

15. López, G. M.; Torres, G. E., et al. (2016). The evolution of Colombian industry in the context of the energy-mining auge: Symptoms of the Dutch disease? Cuadernos de Economia, 35(68), 237-259.

16. Mehlum, H.; Moene, K. et al. (2006). Institutions and the resource curse. Economic Journal, 116(508), 1-20.

17. Neary, J. y Wijnbergen, S. (1986). Natural resources and the macroeconomy: A theoretical framework. Cambridge, Massachusetts: MIT Press.

18. Nyatepe-C. A. (1994). Dutch disease, government policy and import demand in Nigeria. Applied Economics, 26(4), 327-336.

19. Robinson, J. A.; Torvik, R., et al. (2006). Political foundations of the resource curse. Journal of Development Economics, 79(2), 447-468.

20. Rudd, D. (1996). An empirical analysis of Dutch disease: Developing and developed countries. Honors Projects. [http://digitalcommons.iwu. edu/econ_honproj/62].

21. Sachs, J. y Warner, A. (1995). Economic reform and the process of global integration. Brookings Papers on Economic Activity 1995(1), 1-118.

22. Seers, D. (1964). The mechanism of an open petroleum economy. Social and Economic Studies, 13(2), 233-242.

23. Spatafora, N. y Warner, A. (1999). Macroeconomic and sectoral effects of terms of trade shocks. The experience of the oil exporting developing countries. IMF working paper 99(134), 1-56.

24. Stijns, J. P. (2003). An empirical test of the Dutch disease hypothesis using a gravity model of trade. Congress of the EEA, Stocolmo.

25. Uribe, J. y Ortiz, C. (2006). Informalidad laboral en Colombia 19882000. Evolución, teorias y modelos. Cali: Universidad del Valle.

26. Wikstrôm, D. y Ôsterberg, H. (2003). The Dutch disease in a small economy. The case of Jamaica. [http://epubl.1tu.se/1404-5508/2003/024/ LTU-SHU-EX-03024-SE.pdf]. 